v. 9, n. 2 , p. $22-29,2020$

ISSN 2237-9223

\begin{tabular}{l} 
REVISTA BRASIIEIRA DE \\
CRIMINALISTICA \\
\hline
\end{tabular}

DOI: http://dx.doi.org/10.15260/rbc.v9i2.317

\title{
Análise de Pescado Sem Cabeça
}

\author{
A.R. Correia ${ }^{a,}{ }^{*}$, C.R. De Musis ${ }^{\text {a }}$, J.P.N. Filho ${ }^{b}$, M.V.B.R. Ribeiro ${ }^{c}$, S.E.M. Leite ${ }^{\text {c }}$, V.H.M. \\ Danelichen ${ }^{\mathrm{c}}$ \\ ${ }^{a}$ Universidade Federal de Estado de Mato Grosso, Cuiabá (MT), Brasil \\ ${ }^{b}$ Pericia Oficial e Identificação Técnica-POLITEC, Cuiabá (MT), Brasil \\ ${ }^{c}$ Universidade de Cuiabá, Cuiabá (MT), Brasil \\ *Endereço de e-mail para correspondência: antonioramoseng@terra.com.br. Tel.: +55-65-999779294.
}

Recebido em 04/09/2018; Revisado em 05/04/2019; Aceito em 02/06/2020

\begin{abstract}
Resumo
Pescadores e comerciantes de peixes na região da baixada cuiabana desenvolvem diversas estratégias para dificultar a produção de provas de pesca predatória no Estado de Mato Grosso, em especial para o pescado das espécies Cachara (Pseudoplatystoma fasciatum) e Pintado (Pseudoplatystoma corruscans).Essas duas espécies agrupam qualidades inéditas do ponto de vista gastronômico e, por essa razão, possuem grande lucratividade. Como a Lei Estadual n ${ }^{\circ} 9.096$ de 16 de janeiro de 2009 pró́be a captura de pescado por meio de diversos tipos de petrechos, em especial os específicos do artigo 25, item "V" letra "f", que trata sobre os petrechos de malha (rede e tarrafão), os contraventores escolhem a extração das cabeças dos animais no intuito de polemizar o método da captura e ainda o tamanho do pescado. Este trabalho estabelece um modelo estatístico para essas espécies de forma a reconstituir o tamanho do pescado apreendidosem a cabeça, viabilizando a estimativa pericial.
\end{abstract}

Palavras-Chave: Perícia Ambiental; Pescado; Cachara; Pintado.

\section{Abstract}

Fishermen and fish traders in the Cuiabana lowland region have developed several strategies to hinder the production of predatory fishing in the state of Mato Grosso, especially for fish of the species: Cachara (Pseudoplatystoma fasciatum) and; Painted (Pseudoplatystoma corruscans). These two species unite gastronomic qualities and, for this reason, have great profitability. As State Law 9096 of January 16, 2009 prohibits the capture of fish through various types of gear, in particular those specified in article 25, item "V", letter "f", which deals with knitted and crocheted gear), the offenders choose the extraction of the heads of the animals in order to argue the method of capture and the size of the fish. The target of this article is establishing a statistical model for these species in order to reconstitute the size of the fish seized even without the head, making feasible the expert estimate.

Keywords: Environmental Expertise; Fish; Cachara; Pintado.

\section{INTRODUÇÃO}

A necessidade de comprovação da modalidade de captura de pescado, associado à conferência do tamanho dos exemplares apreendidos conflita com as providencias dos comerciantes e pescadores infratores que providenciam toda sorte de meios para driblar ou confundir as autoridades de fiscalização. As espécies mais solicitadas por consumidores da região da baixada cuiabana e, portanto, mais lucrativas são o Cachara (Pseudoplatystoma fasciatum) e o Pintado (Pseudoplatystoma corruscans), espécies de grande valor nutritivo e oriundas das águas da bacia do Rio Paraguai (SOUZA, 2015). Constantemente, os infratores retiram as cabeças dos exemplares capturados por equipamentos de malha para polemizar o tipo de captura nos tribunais, bem como para dissimular o tamanho mínimo daqueles exemplares capturados fora da medida. As autoridades ficam então vinculadas a massa total de 125 $\mathrm{kg}$ permitida por pescador, Lei $\mathrm{n}^{\circ} 9.096$ de 16 de janeiro de 2009) e ao período proibitivo de pesca, sujeitando-se às questões jurídicas dos advogados de defesa dos infratores, 
quanto ao modo de captura e medida mínima do pescado que passam a ser polemica nos tribunais.

Este trabalho, teve como objetivo elaborar um modelo estatístico para predição do tamanho natural do pescado a partir de medidas do corpo do exemplar sem cabeça, conforme ocorre na grande maioria das vezes das apreensões pela fiscalização dos órgãos públicos.

A casuística estudada partiu de pescado associado a pesca predatória obtido nas perícias efetuadas pela Perícia Oficial e Identificação Técnica de Mato Grosso (POLITEC) e no Mercado Municipal de Cuiabá em 2017 e 2018, de modo a permitir a modelagem do tamanho real deste "in natura". Há que se destacar que o pescado considerado foi apreendido na bacia do Rio Paraguai, descartando os oriundos da bacia do Rio Teles Pires e outras bacias.

Quanto ao modo de captura, tem-se que o pescado sem cabeça, por eliminar os vestígios de malha, é, por si só, um indício compatível com a pesca predatória.

A apreensão das espécies cachara e pintado dentre aquelas espécies de couro são as mais frequentes seguidas pela espécie do jau, (Zungaro zungaro), mais remotamente da jurupoca (Hemisorubim platyrhynchos) e outras similares (KUBITZA, 1998). Essas espécies apresentam carne de coloração clara e textura firme, sabor pouco acentuado, baixo teor de gordura e ausência de espinhos intramusculares (KUBITZA, 1998).

$\mathrm{O}$ pescado aqui analisado é proveniente do meio natural, oriundo de apreensão devido à pesca predatória. Os quarenta e cinco exemplares de cada espécie analisados e submetidos a trato estatístico, obtendo uma tabela de correspondência de tamanho natural a partir da cabeça ou do corpo sem a cabeça.

\section{MATERIAIS E MÉTODOS}

\subsection{Materiais}

Os materiais e equipamentos utilizados para este trabalho, foram balança digital marca Toledo com capacidade para $300 \mathrm{~kg}$, classe de exatidão III, grau de proteção IP 40, registrada no Inmetro sob número 2.877.396-2. Fita métrica esmaltada marca Starret de $5 \mathrm{~m}$ de comprimento modelo TS 34, precisão de $2 \mathrm{~mm}$. Máquina fotográfica Sony modelo cybershot.

\section{2. Área de Estudo}

O estudo consistiu em analisar o pescado oriundo de apreensões pelos órgãos fiscalizadores do Estado de Mato Grosso, em âmbito da Perícia Oficial e Identificação Técnica - POLITEC, órgão central de Perícias vinculado a Secretaria de Segurança Pública do Estado de Mato Grosso e a sua gerência de engenharia legal e meio ambiente, através de dados obtidos em oito ocorrências para pescado eviscerados sem cabeça, cachara (Pseudoplatystoma fasciatum) e pintado (Pseudoplatystoma corruscans). Os dados referentes a exemplares inteiros foram obtidos no mercado municipal, apenas para exemplares oriundos da baixada cuiabana.

Pela Figura 1 é possível visualizar a quantidade de córregos, ribeirões e rios no Estado de Mato Grosso. Observar que na área em que impera a bacia do Rio Paraguai, no centro da região sul do Estado foco de estudo deste artigo

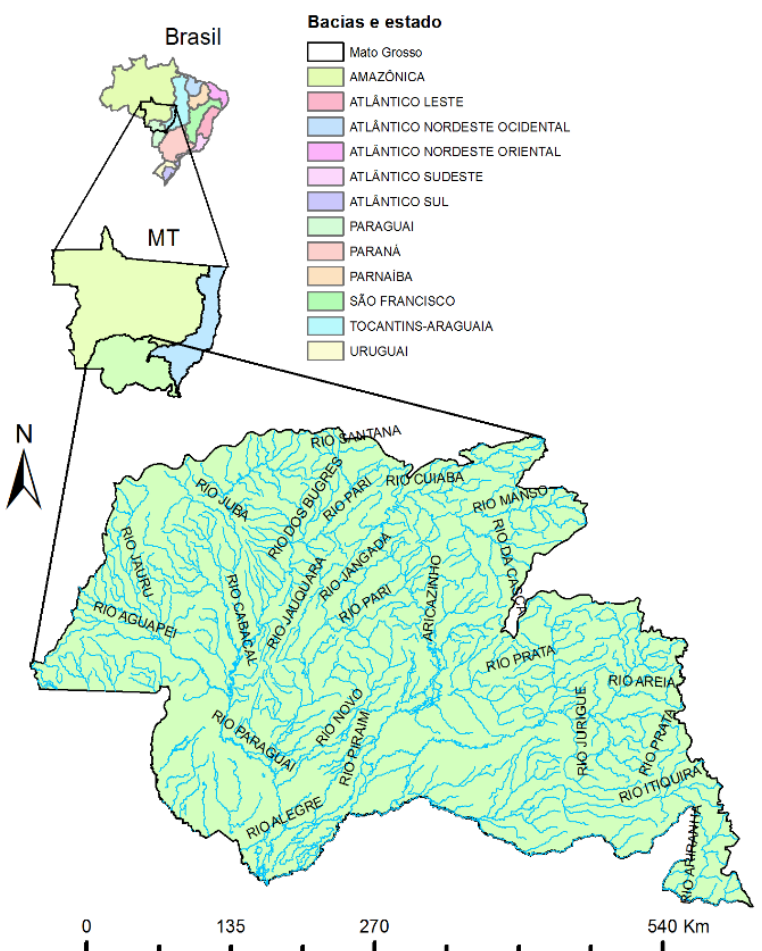

Figura 1. Bacias existentes na região sul de Mato Grosso, sendo a bacia do Paraguai a mais central no mapa.

\subsection{Método}

Através das ocorrências periciais realizadas nos anos de 2017 e 2017 obteve-se parte dos dados de massa e comprimento dos exemplares, complementados por dados obtidos no Mercado de peixe Municipal de Cuiabá, para completar o número de 45 exemplares de cada espécie. A cada lote apreendido e analisado foram registrados comprimento e massa, com e sem cabeça, de exemplares eviscerados, e ainda, retirados um indivíduo de cada espécie para avaliação da carcaça. 


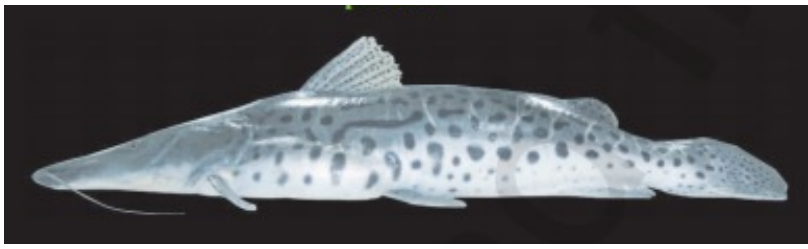

Pseudoplastytoma corruscans (Spix\& Agassiz,1829)

Figura 2. Imagem do Pintado como encontrados na natureza.

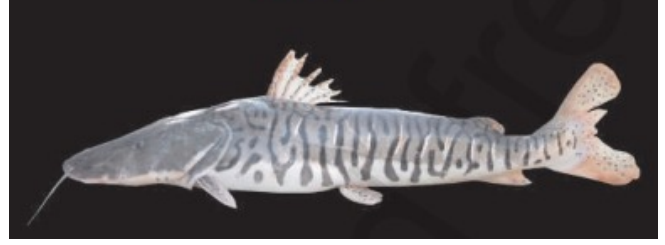

Pseudopatystoma fasciatum (Linnacus, 1766)

Figura 3. Imagem do Cachara como encontrados na natureza.

Tabela 1. Relação peso comprimento dos cachara e pintado em diferentes estudos.

\begin{tabular}{|c|c|c|c|}
\hline Espécie & Macho & Fêmea & Citação \\
\hline Pseudoplatystoma coruscans & $\mathrm{Pt}=2,75^{*} 10^{-6} \mathrm{Cp}^{3,2432}$ & $\mathrm{Pt}=2,05 * 10^{-6} \mathrm{Cp}^{3,2874}$ & Resende et al. $(1995)^{1}$ \\
\hline PseudoplatystomacCoruscans & Pt $0,0019 \mathrm{Cp}^{3,523}$ & --------- & Miranda \& Ribeiro $(1997)^{2}$ \\
\hline Pseudoplatystoma coruscanss & \multicolumn{2}{|c|}{$\mathrm{Pt}=0,00624 \mathrm{Cp}^{3,128}$} & Mateus $(1996)^{2}$ \\
\hline Pseudoplatystoma coruscans & \multicolumn{2}{|c|}{$\mathrm{Pt}=0,001734 \mathrm{Ct}^{3,335}$} & Godinho et al. $(1997)^{2}$ \\
\hline Pseudoplatystoma coruscans & $\mathrm{Pt}=9,30 * 10^{-6} \mathrm{Cp}^{3,06}$ & $\mathrm{Pt}=0,82 * 10^{-6} \mathrm{Cp}^{3,45}$ & Resende et al. $(1995)^{1}$ \\
\hline Pseudoplatystoma fasciatum & $\mathrm{Pt}-0,0032 \mathrm{Ct}^{3,209}$ & $\mathrm{Pt}=0,0014 \mathrm{Ct}^{3,439}$ & Romagosa et al. $(2000)^{2}$ \\
\hline Pseudoplatystoma fasciatum & $\mathrm{Pt}=0,0045 \mathrm{Ct}^{3,13}$ & $\mathrm{Pt}=0,0012 \mathrm{Ct}^{3,45}$ & Romagosa et al. $(2002)^{2}$ \\
\hline
\end{tabular}

$\mathrm{Pt}$ - Peso Total; $\mathrm{Ct}$ - Comprimento total (distância entre o focinho e a ponta da nadadeira caudal); $\mathrm{Cp}$ - Comprimento padrão

(distância entre o focinho e a base de inserção da nadadeira caudal).

${ }^{1}$ Peso em gramas e comprimento e milímetros.

${ }^{2}$ Peso em gramas e comprimento e centímetros.

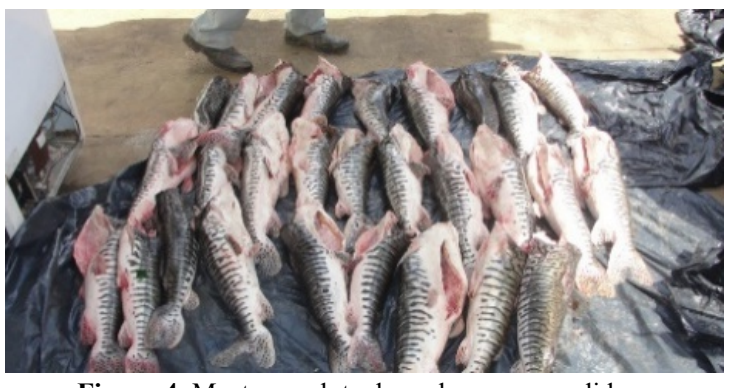

Figura 4. Mostra um lote de cacharas apreendidos.

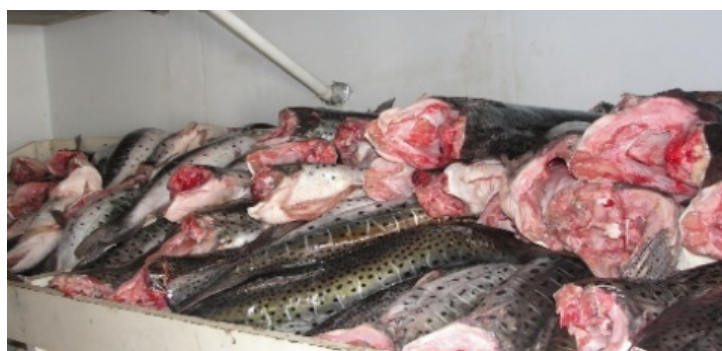

Figura 5. Mostra um lote de pintados apreendidos, ambos eviscerados e sem cabeça peças motivo de perícia. (Foram pesados e medidos 45 exemplares)

\section{RESULTADOS}

\subsection{A análise de pescados sem cabeça provenientes de apreensões}

Foram aferidas massas e medidas do corpo sem cabeça objetos de apreensões policiais da Delegacia de Meio ambiente - DEMA, periciados pela Perícia Oficial e Identificação Técnica - POLITEC, cujo procedimento é pesar, medir, fotografar e descrever o local de apreensão. Os espécimes de cachara e pintado são muito parecidas e, portanto, os dados semelhantes, demonstraram por consequência resultados muito próximos (Figura 1). Ao analisarmos os diagramas de dispersão foi possível perceber para as duas espécies as tendências de tamanhos e pesos para aqueles pescados oriundos de perícias (eviscerados e sem cabeça), demonstrando uma força de associação para essas duas variáveis (menor dispersão) no intervalo de peso até $6 \mathrm{~kg}$ e comprimento do corpo é de até $75 \mathrm{~cm}$. Esse resultado é bastante normal porque os indivíduos das duas espécies têm homogeneidade de peso e comprimento por caracterizarem em indivíduos jovens.
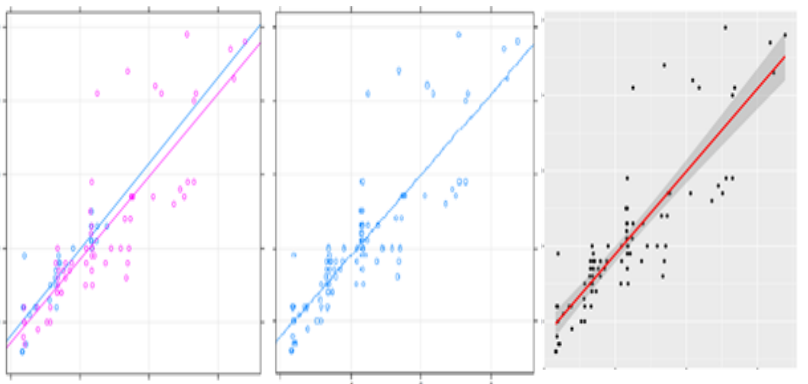

Figura 6. Análise de Dispersão das Variáveis de pescado eviscerados e sem cabeça. 6A-Dispersão considerando as duas espécies; 6B - Linha de tendência para as duas variáveis; 6C-Intervalo de Confiança comum as duas espécies. Legenda da figura está muito pequena. Não é possível ler.

Importante também a análise dos coeficientes linear e angular dos dados dos pescados que representam mais uma comprovação dos tamanhos mínimos de pescado dando segurança a aferição por modelo matemático de regressão simples. Quando examinamos os dois coeficientes mantem a coerência da concentração dos dados entre pescados menores e a dispersão dos maiores (Figura 7). 
Tabela 2. Similaridade de Tamanho das duas espécies.

\begin{tabular}{|c|c|c|c|c|c|}
\hline \multicolumn{6}{|c|}{ (formula = Inteiro Corpo + Espécie + Corpo $*$ Espécie, data = peixe) } \\
\hline \multirow[t]{2}{*}{ Resíduos: } & Min & 10 & Mediana & $3 Q$ & Max \\
\hline & -5.1661 & -1.0334 & 0.2317 & 1.1440 & 3.4181 \\
\hline \multirow[t]{5}{*}{$\begin{array}{l}\text { Detectamos diferença significativa entre Cachara e } \\
\text { Pintado }\end{array}$} & Coeficientes: & Estimado & $\begin{array}{l}\text { Erro } \\
\text { padrão }\end{array}$ & t value & $\operatorname{Pr}(>|t|)$ \\
\hline & (Intercepto) & 5.02585 & 2.14149 & 2.347 & 0.0225 \\
\hline & Corpo & 1.32700 & 0.03332 & 39.822 & $<2 \mathrm{e}-16$ \\
\hline & Especie1 & 5.27412 & 2.14149 & 2.463 & 0.0169 \\
\hline & Corpo:Especie1 & 0.07348 & 2.14149 & -2.205 & 0.0316 \\
\hline \multicolumn{6}{|c|}{ Signif. : $0^{\prime * * * \prime} 0.001^{\prime * * \prime} 0.01^{\prime * \prime} 0.05^{\prime \prime} 0.1^{\prime \prime} 1$} \\
\hline \multicolumn{6}{|c|}{ Resíduo do erro padrão: 1.812 em 56 graus de liberdade } \\
\hline \multicolumn{6}{|c|}{ R quadrado múltiplo: 0.9882, R quadrado ajustado: 0.9876} \\
\hline \multicolumn{6}{|c|}{ F-statistic: 1563 em 3 and 56 DF, p-value: $<2.2 \mathrm{e}-16$} \\
\hline
\end{tabular}

Tabela 3a. Relação peso comprimento do cachara.

\begin{tabular}{|c|c|c|c|c|c|}
\hline \multicolumn{6}{|c|}{ (formula = Inteiro $\sim$ Corpo, data = cachara) } \\
\hline \multirow[t]{2}{*}{ Resíduos: } & Min & $1 Q$ & Mediana & $3 Q$ & Max \\
\hline & -5.1661 & 0.5257 & 0.3585 & 0.8522 & 2.4888 \\
\hline \multirow[t]{3}{*}{ Regressão para o Cachara } & Coeficientes: & Estimado & Erro padrão & t value & $\operatorname{Pr}(>|t|)$ \\
\hline & (Intercepto) & 10.29997 & 3.68425 & 2.796 & 0.00925 \\
\hline & Corpo & 1.25352 & 0.05852 & 21.419 & $<2 \mathrm{e}-16$ \\
\hline \multicolumn{6}{|c|}{ Códigos: $0^{\prime * * * \prime} 0.001^{\prime * * \prime} 0.01^{\prime * \prime} 0.05^{\prime \prime} .0 .1^{\prime \prime} 1$} \\
\hline \multicolumn{6}{|c|}{ Resíduos do erro padrão: 1.696 em 28 graus de liberdade } \\
\hline \multicolumn{6}{|c|}{ R quadrado múltiplo: 0.9425, R quadrado ajustado: 0.9404} \\
\hline \multicolumn{6}{|c|}{ F-statistic: 458.8 em 1 and 28 DF, p-value: $<2.2 \mathrm{e}-16$} \\
\hline cemfint(mod_c) & $2.5 \%$ & $97.5 \%$ & & & \\
\hline (Intercepto) & 2.753127 & 17.846815 & & & \\
\hline Corpo & 1.133640 & 1.373402 & & & \\
\hline
\end{tabular}

Tabela 3b. Relação peso comprimento do pintado.

\begin{tabular}{|c|c|c|c|c|c|}
\hline \multicolumn{6}{|c|}{ (formula = Inteiro $\sim$ Corpo, data = pintado) } \\
\hline \multirow[t]{2}{*}{ Resíduos: } & Min & 10 & Mediana & $3 Q$ & Max \\
\hline & -4.0019 & -1.4903 & 0.0225 & 1.4102 & 3.4181 \\
\hline \multirow[t]{3}{*}{ Regressão para o Pintado } & Coeficientes: & Estimado & Erro padrão & t value & $\operatorname{Pr}(>|t|)$ \\
\hline & (Intercepto) & -0.24827 & 1.78776 & -0.139 & 0.891 \\
\hline & Corpo & 1.40049 & 0.02442 & 57.348 & $<2 \mathrm{e}-16$ \\
\hline \multicolumn{6}{|c|}{ Códigos: $0^{\prime * * * \prime} 0.001^{\prime * * \prime} 0.01^{\prime * \prime} 0.05^{\prime \prime} .0 .1^{\prime \prime} 1$} \\
\hline \multicolumn{6}{|c|}{ Resíduos do erro padrão: 1.922 em 28 graus de liberdade } \\
\hline \multicolumn{6}{|c|}{ R quadrado múltiplo: 0.9916, R quadrado ajustado: 0.9913} \\
\hline \multicolumn{6}{|c|}{ F-statistic: 3289 em 1 and 28 DF, p-value: $<2.2 \mathrm{e}-16$} \\
\hline (mod_p) & $2.5 \%$ & $97.5 \%$ & & & \\
\hline (Intercepto) & -3.910324 & 3.413789 & & & \\
\hline Corpo & 1.350463 & 1.450510 & & & \\
\hline
\end{tabular}




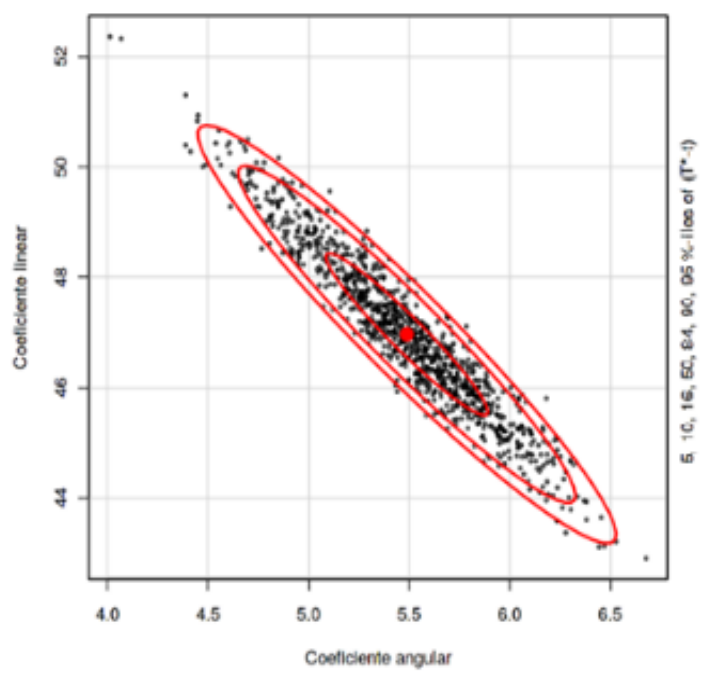

Figura 7. Uma visão dos coeficientes angular e linear.

\subsection{Análise de pescado observado no mercado de peixes em Cuiabá.}

As apreensões de pescado objeto deste trabalho demandaram em sua maioria responder a autoridade requisitante se os indivíduos da apreensão estavam enquadrados na medida mínima prevista pela Lei $\mathrm{n}^{\circ}$ 9.893/2013.

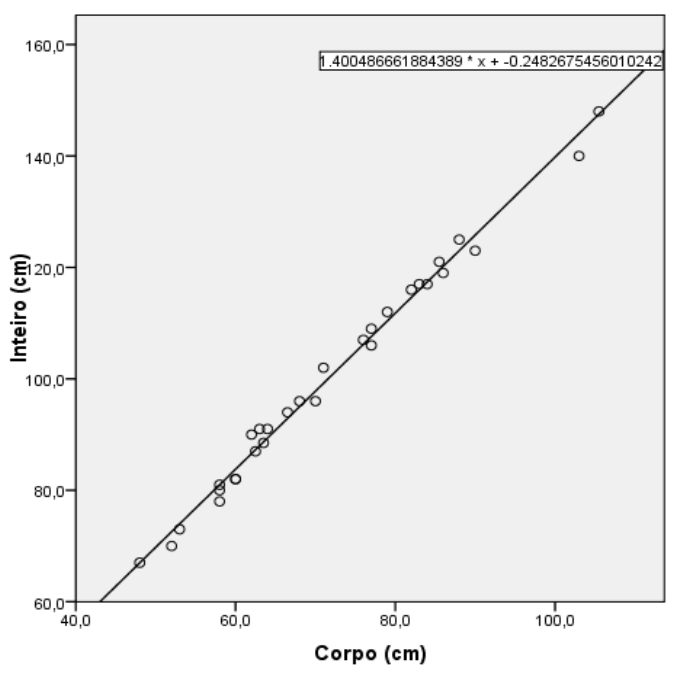

Figura 8. Diagrama de dispersão para o quadro 02 - Pintados. Peixe em tamanho natural X Peixe sem cabeça.

O Quadro 2 (Pintados) e Quadro 3 (Cacharas) e respectivos diagramas demonstram a relação entre comprimento total observado, tamanho da cabeça observado e tamanho do corpo sem cabeça observado, de cientifica às demandas judiciais correntes.

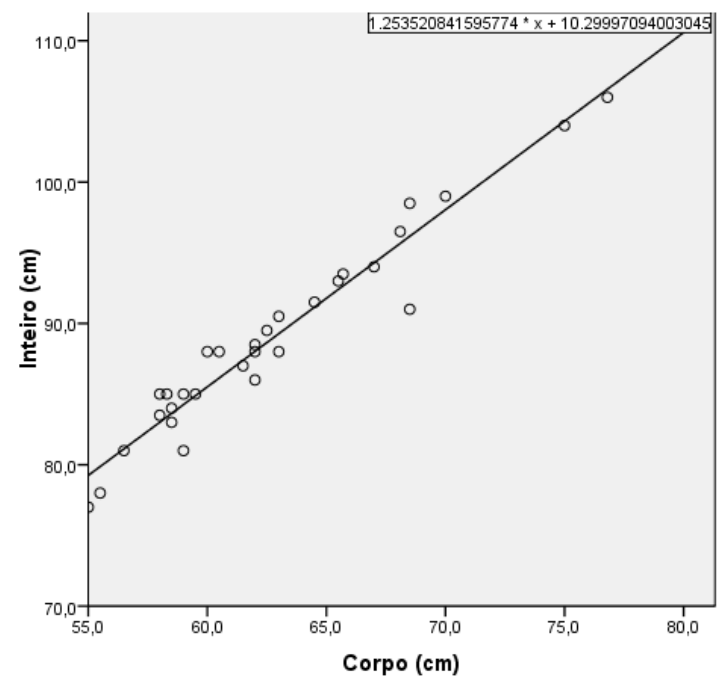

Figura 9. Diagrama de dispersão para o quadro 03 - Cacharas.

As equações preditivas a partir do corpo sem cabeça para as espécies foram:

\section{Cachara (Pseudopatystoma fasciatum)} $1,2535 \mathrm{X}+10,2999$

Onde o valor do coeficiente angular é 1,2535 denominado como $\beta$ e o coeficiente linear é 10,2999 denominado como intercepto. A planilha abaixo em Excel é um mecanismo pronto para a predição do comprimento total do corpo a partir do corpo sem cabeça. Para este caso com valores preenchidos para o corpo sem cabeça e o valor estimado do comprimento total do peixe.

\section{Pintado (Pseudoplastytoma corruscans) 1,4004 X $-0,2482$}

Onde o valor do coeficiente angular é 1,4004 denominado como $\beta$ e o coeficiente linear é -0,2482) denominado como $\beta$. A planilha abaixo (tabela 4 b) é um mecanismo pronto para a predição do comprimento total do corpo a partir do corpo sem cabeça. Neste caso para demonstração sem valores preenchidos para o corpo sem cabeça.

As equações preditivas a partir apenas da cabeça para as espécies foram:

\section{Cachara (Pseudopatystoma fasciatum) $0,2463 \mathrm{X}+4,29$}

Onde o valor do coeficiente angular é 0,2463 denominado como $\beta$ e o coeficiente linear é 4,29 denominado como $\alpha$. Para predição do tamanho total do peixe a partir do tamanho somente da cabeça caso em que as vezes em perícia ou outra ação policial em frigoríficos encontra apenas a cabeça. Mesmo raciocínio para a espécie do pintado: 
Pintado (Pseudoplastytoma corruscans)

$$
0,292 \mathrm{X}+(-0,7818)
$$

Onde o valor do coeficiente angular é 0,292 denominado como $\beta$ e o coeficiente linear é $-0,7818$ denominado como $\beta$.

\section{CONCLUSÕES}

O problema de captura de pescado através de pesca ilegal é constante em Mato Grosso, devido a abundância de espécies e exemplares de elevado valor nutricional e de agradável paladar. As espécies Cachara e Pintado são por excelência enquadrados nessas condições, sendo o objeto principal no mercado de peixes em todo estado. O presente trabalho conclui que:

1 - Foi possível estimar o tamanho total dos peixes capturados fora da lei e apreendidos pelo sistema de Segurança Pública, a partir do tamanho dos exemplares sem cabeça;

2 - Foi possível estimar o tamanho total dos peixes capturados fora da lei e apreendidos pelo sistema de Segurança Pública, a partir do tamanho da cabeça.

3 - É possível estimar o tamanho total dos peixes a partir do comprimento das peças de suam (coluna vertebral completa com a nadadeira de cauda) que esteja inteiro em seu comprimento;

Válido igual ao item 1 para as duas espécies de pescado.

O modelo estatístico permite argumentação jurídica de boa aceitação, uma vez que os resultados aqui obtidos estão coerentes com as pesquisas cientificas elencadas na bibliografia anexada. Como resultado secundário, em consequência da volumosa quantidade de peixes apreendido, sugere adoção da mesma política adotada pela lei 9893/2013 proibitiva a pesca do dourado, estender também a pesca do cachara e pintado para evitar que estas duas espécies entrem em risco de serem extintas. A proibição de pesca do Dourado (Salminus brasiliensis) operou resultados visíveis para qualquer observador nos rios da região especialmente na região de Barão de Melgaço próximo as baias de Sia mariana e Chacororé.

\section{AGRADECIMENTOS}

Nosso agradecimento aos companheiros da Politec, aos delegados e investigadores da Delegacia Especial de Meio Ambiente - DEMA, ao Programa de Pós-Graduação em Física Ambiental - PGFA, que apoiaram a iniciativa. Agradecemos também os comerciantes de pescado da feira do Porto, nas pessoas do Sr. Aroldo Rodrigues de Souza do box de vendas $n^{\circ} 5$ e ao Sr. João Santana Correia do box 18 pela gentileza em autorizar as medições do pescado.

\section{REFERÊNCIAS BIBLIOGRÁFICAS}

[1]CHAVES, G.V. Técnica de videoceloscopia para sexagem em surubim. Escola de veterinaria da UFMG 2011.

[2]LAUDOS PERICIAIS DA POLITEC - Gerencia de pericias em engenharia legal e meio ambiente - 2017.

[3]LIRANÇO, A.D.S et al; Desempenho produtivo de Pseudoplatystoma corruscans estocados em sistemas de criação: semi-intensivo(viveiro escavado) e intensivo (tanque rede) - Ciencia Rural, Santa Maria, 2011 - ISSN 0103-8478

[4]ROMAGOSA, E. et al; Biologia reprodutiva de fêmeas de cachara, Pseudoplatystoma fasciatum [5](TELEOSTEI, SILURIFORMES, PIMELODIDAE), mantidas em cativeiro. Instituto de Pesca São Paulo - 2004.

[6]ROMAGOSA, E. et al; característicasmorfometricas e crescimento do cachara, Pseudoplatystoma fasciatum (linnaeus, 1766), e cativeiro - Instituto de Pesca, APTA, SAA, São Paulo, 2016.

[7]ROTTA, M. A; Ictiometro para biometria de surubins (pintado e cachara). Comunicado Técnico 28 - ISSN 1517-4875 Corumbá, MS, 2003. 
Tabela 4a. Valores preditivos a partir do corpo sem cabeça para o Cachara.

\begin{tabular}{|c|c|c|c|}
\hline \multicolumn{4}{|c|}{ CACHARA } \\
\hline $\begin{array}{l}\text { Comprimento } \\
\text { Corpo }(\mathrm{cm})\end{array}$ & $\beta$ & $\alpha$ & $\begin{array}{c}\text { Comprimento total } \\
\text { estimado }(\mathrm{cm})\end{array}$ \\
\hline 40 & 1,253521 & 10,29997 & 60,44081 \\
\hline 41 & 1,253521 & 10,29997 & 61,694331 \\
\hline 42 & 1,253521 & 10,29997 & 62,947852 \\
\hline 43 & 1,253521 & 10,29997 & 64,201373 \\
\hline 44 & 1,253521 & 10,29997 & 65,454894 \\
\hline 45 & 1,253521 & 10,29997 & 66,708415 \\
\hline 46 & 1,253521 & 10,29997 & 67,961936 \\
\hline 47 & 1,253521 & 10,29997 & 69,215457 \\
\hline 48 & 1,253521 & 10,29997 & 70,468978 \\
\hline 49 & 1,253521 & 10,29997 & 71,722499 \\
\hline 50 & 1,253521 & 10,29997 & 72,97602 \\
\hline 51 & 1,253521 & 10,29997 & 74,229541 \\
\hline 52 & 1,253521 & 10,29997 & 75,483062 \\
\hline 53 & 1,253521 & 10,29997 & 76,736583 \\
\hline 54 & 1,253521 & 10,29997 & 77,990104 \\
\hline 55 & 1,253521 & 10,29997 & 79,243625 \\
\hline 56 & 1,253521 & 10,29997 & 80,497146 \\
\hline 57 & 1,253521 & 10,29997 & 81,750667 \\
\hline 58 & 1,253521 & 10,29997 & 83,004188 \\
\hline 59 & 1,253521 & 10,29997 & 84,257709 \\
\hline 60 & 1,253521 & 10,29997 & 85,51123 \\
\hline 61 & 1,253521 & 10,29997 & 86,764751 \\
\hline 62 & 1,253521 & 10,29997 & 88,018272 \\
\hline 63 & 1,253521 & 10,29997 & 89,271793 \\
\hline 64 & 1,253521 & 10,29997 & 90,525314 \\
\hline 65 & 1,253521 & 10,29997 & 91,778835 \\
\hline 66 & 1,253521 & 10,29997 & 93,032356 \\
\hline 67 & 1,253521 & 10,29997 & 94,285877 \\
\hline 68 & 1,253521 & 10,29997 & 95,539398 \\
\hline 69 & 1,253521 & 10,29997 & 96,792919 \\
\hline 70 & 1,253521 & 10,29997 & 98,04644 \\
\hline 71 & 1,253521 & 10,29997 & 99,299961 \\
\hline 72 & 1,253521 & 10,29997 & 100,553482 \\
\hline 73 & 1,253521 & 10,29997 & 101,807003 \\
\hline 74 & 1,253521 & 10,29997 & 103,060524 \\
\hline 75 & 1,253521 & 10,29997 & 104,314045 \\
\hline 76 & 1,253521 & 10,29997 & 105,567566 \\
\hline 77 & 1,253521 & 10,29997 & 106,821087 \\
\hline 78 & 1,253521 & 10,29997 & 108,074608 \\
\hline 79 & 1,253521 & 10,29997 & 109,328129 \\
\hline 80 & 1,253521 & 10,29997 & 110,58165 \\
\hline 81 & 1,253521 & 10,29997 & 111,835171 \\
\hline 82 & 1,253521 & 10,29997 & 113,088692 \\
\hline 83 & 1,253521 & 10,29997 & 114,342213 \\
\hline 84 & 1,253521 & 10,29997 & 115,595734 \\
\hline 85 & 1,253521 & 10,29997 & 116,849255 \\
\hline
\end{tabular}

Tabela 4b. Valores preditivos a partir do corpo sem cabeça para o Pintado.

\begin{tabular}{|c|c|c|c|}
\hline \multicolumn{4}{|c|}{ PINTADO } \\
\hline $\begin{array}{l}\text { Comprimento } \\
\text { Corpo }(\mathrm{cm})\end{array}$ & $\beta$ & $\alpha$ & $\begin{array}{c}\text { Comprimento total } \\
\text { estimado }(\mathrm{cm})\end{array}$ \\
\hline 40 & 1,400487 & 0,2482675 & 56,2677475 \\
\hline 41 & 1,400487 & 0,2482675 & 57,6682345 \\
\hline 42 & 1,400487 & 0,2482675 & 59,0687215 \\
\hline 43 & 1,400487 & 0,2482675 & 60,4692085 \\
\hline 44 & 1,400487 & 0,2482675 & 61,8696955 \\
\hline 45 & 1,400487 & 0,2482675 & 63,2701825 \\
\hline 46 & 1,400487 & 0,2482675 & 64,6706695 \\
\hline 47 & 1,400487 & 0,2482675 & 66,0711565 \\
\hline 48 & 1,400487 & 0,2482675 & 67,4716435 \\
\hline 49 & 1,400487 & 0,2482675 & 68,8721305 \\
\hline 50 & 1,400487 & 0,2482675 & 70,2726175 \\
\hline 51 & 1,400487 & 0,2482675 & 71,6731045 \\
\hline 52 & 1,400487 & 0,2482675 & 73,0735915 \\
\hline 53 & 1,400487 & 0,2482675 & 74,4740785 \\
\hline 54 & 1,400487 & 0,2482675 & 75,8745655 \\
\hline 55 & 1,400487 & 0,2482675 & 77,2750525 \\
\hline 56 & 1,400487 & 0,2482675 & 78,6755395 \\
\hline 57 & 1,400487 & 0,2482675 & 80,0760265 \\
\hline 58 & 1,400487 & 0,2482675 & 81,4765135 \\
\hline 59 & 1,400487 & 0,2482675 & 82,8770005 \\
\hline 60 & 1,400487 & 0,2482675 & 84,2774875 \\
\hline 61 & 1,400487 & 0,2482675 & 85,6779745 \\
\hline 62 & 1,400487 & 0,2482675 & 87,0784615 \\
\hline 63 & 1,400487 & 0,2482675 & 88,4789485 \\
\hline 64 & 1,400487 & 0,2482675 & 89,8794355 \\
\hline 65 & 1,400487 & 0,2482675 & 91,2799225 \\
\hline 66 & 1,400487 & 0,2482675 & 92,6804095 \\
\hline 67 & 1,400487 & 0,2482675 & 94,0808965 \\
\hline 68 & 1,400487 & 0,2482675 & 95,4813835 \\
\hline 69 & 1,400487 & 0,2482675 & 96,8818705 \\
\hline 70 & 1,400487 & 0,2482675 & 98,2823575 \\
\hline 71 & 1,400487 & 0,2482675 & 99,6828445 \\
\hline 72 & 1,400487 & 0,2482675 & 101,0833315 \\
\hline 73 & 1,400487 & 0,2482675 & 102,4838185 \\
\hline 74 & 1,400487 & 0,2482675 & 103,8843055 \\
\hline 75 & 1,400487 & 0,2482675 & 105,2847925 \\
\hline 76 & 1,400487 & 0,2482675 & 106,6852795 \\
\hline 77 & 1,400487 & 0,2482675 & 108,0857665 \\
\hline 78 & 1,400487 & 0,2482675 & 109,4862535 \\
\hline 79 & 1,400487 & 0,2482675 & 110,8867405 \\
\hline 80 & 1,400487 & 0,2482675 & 112,2872275 \\
\hline 81 & 1,400487 & 0,2482675 & 113,6877145 \\
\hline
\end{tabular}


Tabela $4 b$ (cont.). Valores preditivos a partir do corpo sem cabeça para o Pintado.

\begin{tabular}{c|c|c|c}
\hline \multicolumn{5}{c}{ PINTADO } \\
\hline $\begin{array}{c}\text { Comprimento } \\
\text { Corpo (cm) }\end{array}$ & $\beta$ & $\alpha$ & $\begin{array}{c}\text { Comprimento total } \\
\text { estimado (cm) }\end{array}$ \\
\hline 82 & 1,400487 & 0,2482675 & 115,0882015 \\
\hline 83 & 1,400487 & 0,2482675 & 116,4886885 \\
\hline 84 & 1,400487 & 0,2482675 & 117,8891755 \\
\hline 85 & 1,400487 & 0,2482675 & 119,2896625 \\
\hline
\end{tabular}

Tabela 5a. Valores preditivos para o Cachara a partir do comprimento da cabeça.

\begin{tabular}{|c|c|c|c|}
\hline \multicolumn{4}{|c|}{ CACHARA } \\
\hline $\begin{array}{l}\text { Comprimento Cabeça } \\
(\mathrm{cm})\end{array}$ & $\beta$ & $\alpha$ & $\begin{array}{c}\text { Comprimento total } \\
\text { estimado }(\mathrm{cm})\end{array}$ \\
\hline 15 & 0,2463 & 4,29 & 79,845 \\
\hline 16 & 0,2463 & 4,29 & 82,308 \\
\hline 17 & 0,2463 & 4,29 & 84,771 \\
\hline 18 & 0,2463 & 4,29 & 87,234 \\
\hline 19 & 0,2463 & 4,29 & 89,697 \\
\hline 20 & 0,2463 & 4,29 & 92,16 \\
\hline 21 & 0,2463 & 4,29 & 94,623 \\
\hline 22 & 0,2463 & 4,29 & 97,086 \\
\hline 23 & 0,2463 & 4,29 & 99,549 \\
\hline 24 & 0,2463 & 4,29 & 102,012 \\
\hline 25 & 0,2463 & 4,29 & 104,475 \\
\hline 26 & 0,2463 & 4,29 & 106,938 \\
\hline 27 & 0,2463 & 4,29 & 109,401 \\
\hline 28 & 0,2463 & 4,29 & 111,864 \\
\hline 29 & 0,2463 & 4,29 & 114,327 \\
\hline 30 & 0,2463 & 4,29 & 116,79 \\
\hline 31 & 0,2463 & 4,29 & 119,253 \\
\hline 32 & 0,2463 & 4,29 & 121,716 \\
\hline 33 & 0,2463 & 4,29 & 124,179 \\
\hline 34 & 0,2463 & 4,29 & 126,642 \\
\hline 35 & 0,2463 & 4,29 & 129,105 \\
\hline 36 & 0,2463 & 4,29 & 131,568 \\
\hline 37 & 0,2463 & 4,29 & 134,031 \\
\hline 38 & 0,2463 & 4,29 & 136,494 \\
\hline 39 & 0,2463 & 4,29 & 138,957 \\
\hline 40 & 0,2463 & 4,29 & 141,42 \\
\hline
\end{tabular}

Tabela 5b. Valores preditivos para o Pintado a partir do comprimento da cabeça.

\begin{tabular}{|c|c|c|c|}
\hline \multicolumn{4}{|c|}{ PINTADO } \\
\hline $\begin{array}{l}\text { Comprimento } \\
\text { Cabeça }(\mathrm{cm})\end{array}$ & $\beta$ & $\alpha$ & $\begin{array}{c}\text { Comprimento total } \\
\text { estimado }(\mathrm{cm})\end{array}$ \\
\hline 15 & 0,292 & $-0,7818$ & 35,982 \\
\hline 16 & 0,292 & $-0,7818$ & 38,902 \\
\hline 17 & 0,292 & $-0,7818$ & 41,822 \\
\hline 18 & 0,292 & $-0,7818$ & 44,742 \\
\hline 19 & 0,292 & $-0,7818$ & 47,662 \\
\hline 20 & 0,292 & $-0,7818$ & 50,582 \\
\hline 21 & 0,292 & $-0,7818$ & 53,502 \\
\hline 22 & 0,292 & $-0,7818$ & 56,422 \\
\hline 23 & 0,292 & $-0,7818$ & 59,342 \\
\hline 24 & 0,292 & $-0,7818$ & 62,262 \\
\hline 25 & 0,292 & $-0,7818$ & 65,182 \\
\hline 26 & 0,292 & $-0,7818$ & 68,102 \\
\hline 27 & 0,292 & $-0,7818$ & 71,022 \\
\hline 28 & 0,292 & $-0,7818$ & 73,942 \\
\hline 29 & 0,292 & $-0,7818$ & 76,862 \\
\hline 30 & 0,292 & $-0,7818$ & 79,782 \\
\hline 31 & 0,292 & $-0,7818$ & 82,702 \\
\hline 32 & 0,292 & $-0,7818$ & 85,622 \\
\hline 33 & 0,292 & $-0,7818$ & 88,542 \\
\hline 34 & 0,292 & $-0,7818$ & 91,462 \\
\hline 35 & 0,292 & $-0,7818$ & 94,382 \\
\hline 36 & 0,292 & $-0,7818$ & 97,302 \\
\hline 37 & 0,292 & $-0,7818$ & 100,222 \\
\hline 38 & 0,292 & $-0,7818$ & 103,142 \\
\hline 39 & 0,292 & $-0,7818$ & 106,062 \\
\hline 40 & 0,292 & $-0,7818$ & 108,982 \\
\hline
\end{tabular}

